\title{
Pericardial, pleural and diaphragmatic endometriosis in association with pelvic peritoneal and bowel endometriosis: a case report and review of the literature
}

\author{
Marcello Ceccaroni ${ }^{1,2}$, Giovanni Roviglione ${ }^{1,2}$, Piergiorgio Rosenberg ${ }^{3}$, Anna Pesci ${ }^{4}$, Roberto Clarizia ${ }^{1,5}$, Francesco Bruni ${ }^{1,2}$ \\ Claudio Zardini ${ }^{6}$, Giacomo Ruffo ${ }^{6}$, Angelo Placci ${ }^{7}$, Stefano Crippa ${ }^{6}$, Luca Minelli² \\ ${ }^{1}$ Gynecologic Oncology Division, International School of Surgical Anatomy, Sacred Heart Hospital, Negrar, Verona, Italy \\ 2Department of Obstetrics and Gynecology, European Gynaecology Endoscopy School, Sacred Heart Hospital, Negrar, Verona, Italy \\ 32Department of Obstetrics and Gynecology, Foggia University Hospital, Foggia, Italy \\ 4Department of Human Pathology, Sacred Heart Hospital, Negrar, Verona, Italy \\ ${ }^{5}$ Department of Obstetrics and Gynecological Sciences, University of Naples “Federico II", Naples, Italy \\ ${ }^{6}$ Department of General Surgery, Sacred Heart Hospital, Negrar, Verona, Italy \\ 7Department of Cardiology, Santa Maria delle Croci Hospital, Ravenna, Italy
}

Videosurgery and Other Miniinvasive Techniques 2012; 7 (2): 122-131 DOI: 10.5114/wiitm.2011.26758

\begin{abstract}
Diaphragmatic endometriosis is a rare entity, often asymptomatic, which has been described only in small series. It is almost always associated with severe pelvic involvement. The most plausible theory about this condition is based on retrograde menstruation and subsequent transportation of viable cells in peritoneal fluid from the pelvis up the right gutter to the right hemidiaphragm, thus demonstrating its asymmetric distribution on the diaphragm. Pre-operative diagnosis is poorly supported by imaging techniques. In most cases, it is an incidental finding because the lesions may hide behind the right hepatic lobe. In that case it cannot be easily demonstrated with a laparoscope from an umbilical port. Symptomatic diaphragmatic endometriosis is associated with deep lesions which can involve the entire thickness of the diaphragm. In these cases, treatment is more difficult with possible incomplete pain relief and a considerable possibility of recurrence. In this subset, abdominal surgery is recommended. Surgical treatment must be individualized on the basis of the patient's age, fertility desires, type and location of disease and symptoms. We report the surgical treatment of a patient with synchronous pericardial, pleural and diaphragmatic endometriosis associated with pelvic peritoneal and bowel involvement. A review of the literature regarding pericardial and diaphragmatic endometriosis focusing on anatomical and surgical aspects of its management is undertaken.
\end{abstract}

Key words: endometriosis, bowel, laparoscopy, diaphragm, pericardium, pleura.

\section{Introduction}

Endometriosis usually occurs in the pelvis and the most commonly involved sites are the ovaries, the uterosacral and broad ligaments and the parietal pelvic peritoneum. However, involvement of extrapelvic organs is not uncommon [1, 2]. Ectopic endometrium has been found in the umbilicus, skin, vagi- 
na, vulva, cervix, and perineum, in the inguinal canal, upper abdominal peritoneum and organs (liver, spleen), gastrointestinal tract, urinary system, breasts, diaphragm, pleural cavity, brain, eye, lymph nodes, lung and pericardium [3-5]. Exceptional cases have also been described in males [6, 7].

Diaphragmatic endometriosis has been described in case reports and in small series [8-18]; it can be asymptomatic or pain can be referred to the right or left upper abdominal quadrants, thorax and arms. In these cases the entire thickness of the muscle can be involved by endometriosis with extension into the pleural space, resulting in pneumo- or haemothorax. More than 100 cases of thoracic endometriosis have been described [3]. However, this condition raises important diagnostic and treatment challenges [19].

To our knowledge, there is no case report describing surgical treatment of pericardial endometriosis. We report the surgical treatment of a patient with synchronous pericardial, pleural and diaphragmatic endometriosis associated with pelvic peritoneal and bowel involvement and a review of the literature about pericardial and diaphragmatic endometriosis including anatomical and surgical aspects of its management.

\section{Case report}

A 28-year-old nulliparous Caucasian female, with clinical history of endometriosis, presented with recurrent cyclic pelvic pain and right upper-quadrant abdominal pain.

The patient reported catamenial radiation of upper right abdominal pain to the right shoulder which worsened during inspiration and when supine, with impaired motility and sensitivity during pain peaks. The electrocardiography (ECG) and the clinical examination of the chest was unremarkable. A pericardial friction rub was not detected. Accordingly, the patient was given non-steroidal anti-inflammatory and analgesic therapy for months but with no pain relief, although there was partially improved shoulder motility. Pain was evaluated using a visual analogue scale (VAS) - a horizontal line, $100 \mathrm{~mm}$ in length, anchored by word descriptors at each end - and the patient was asked to indicate the degree of pain for each parameter ( 1 = absent, 10 = unbearable). The woman reported mild dyschezia, dysmenorrhoea, dyspareunia, dysuria (VAS score $=1$ ), moderate cyclic pelvic pain (VAS score $=6$ ), and refractory severe right upper-abdominal and right shoulder pain (VAS score $=9$ ), suspicious for diaphragmatic and pleural involvement [20].

Six months before, in another institution, she underwent laparoscopy for enucleation of an endometriotic ovarian cyst, at which time diaphragmatic endometriosis was diagnosed. An upper abdominal ultrasound showed two hypoechoic plaques of $13 \mathrm{~mm}$ and $15 \mathrm{~mm}$ on the anterior surface of the liver's left lobe, another subcentimetric plaque on the medial surface of the same lobe and a plaque below the right diaphragm. Pelvic ultrasound showed a hypoechoic plaque of $16 \mathrm{~mm}$ behind the uterine isthmus and normal uterus and ovaries. All these findings were compatible with endometriosis. Double-contrast barium enema revealed a $2-\mathrm{cm}$ nodular infiltration of the external rectal wall, with no apparent mucosal involvement.

Informed consent was obtained for laparoscopic radical excision of endometriosis and possible abdominal surgery for eradication of diaphragmatic disease.

\section{Laparoscopic pelvic phase}

The patient was placed in a dorsal lithotomic position, under general anaesthesia. Initially, a $10 \mathrm{~mm}$ umbilical trocar for the $0^{\circ}$ laparoscope was placed after creation of pneumoperitoneum with a Veress needle. Two $5 \mathrm{~mm}$ ports (suprapubic and left lower quadrant) and an additional $12 \mathrm{~mm}$ port (right lower quadrant) were placed.

Multiple deep nodules (the largest of about $2 \mathrm{~cm}$ ) involved the broad and round ligaments, vesico-uterine peritoneal fold and the right paracolic gutter. The pouch of Douglas was partially obliterated by dense recto-uterine adhesions and a $2 \mathrm{~cm}$ middle-rectal nodule, infiltrating the mesorectum. A 4-cm nodule infiltrated the rectovaginal septum.

The right and left diaphragmatic peritoneum was completely infiltrated by multiple confluent nodules (Figure 1), the largest measuring $5 \mathrm{~cm}$, involving the central tendon of the diaphragm, near the upper hepatic veins and on the diaphragmatic pericardial insertion.

Thick, vascularized hepato-diaphragmatic adhesions extensively involved Glisson's capsule and a $3 \mathrm{~cm}$ nodule involved the hepatic falciform ligament; the round ligament was apparently free of disease. The pelvic phase was firstly conducted by 

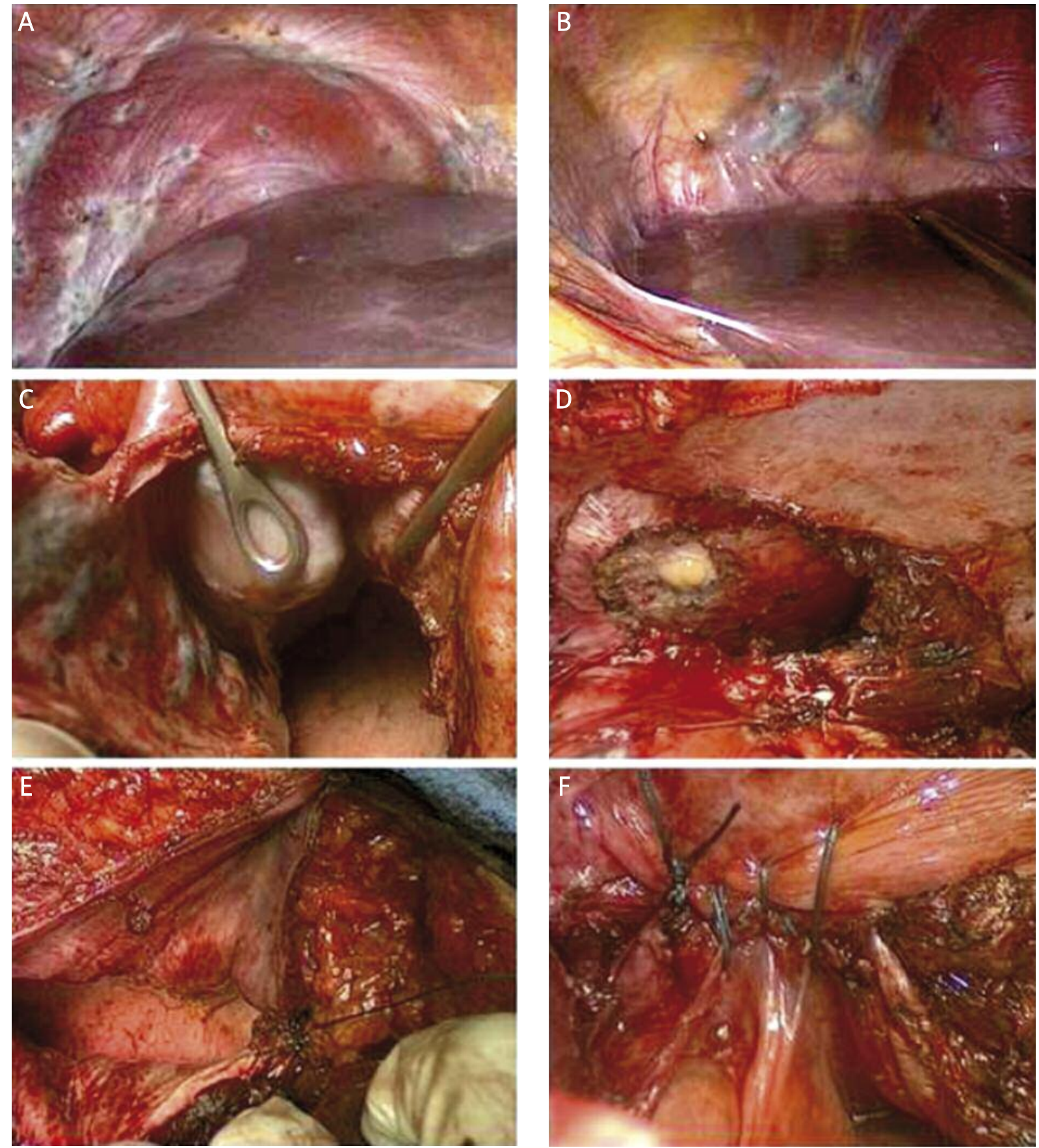

Figure 1. Right (A) and left (B) diaphragmatic peritoneum widely involved by confluent nodules, from $0.5 \mathrm{~cm}$ to $5 \mathrm{~cm}$, infiltrating the diaphragmatic central tendon, close to the upper hepatic veins and on the diaphragmatic pericardial insertion. Right (C) and left (D) wide diaphragmatic resection, with opening of the pleural and pericardial cavity, revealing multiple parietal full-thickness infiltrating nodules involving the parietal pleura, the diaphragmatic central tendon and infiltrating the diaphragmatic side of the pericardium. Right pleural and diaphragmatic suture $(\mathrm{E})$ and left pleural and diaphragmatic suture with pericardial window closure (F) by a single layer (1-0 polypropylene) suture 
a laparoscopic approach, then the hepato-diaphragmatic steps by the abdominal route in order to obtain a whole posterior diaphragmatic evaluation and radical excision of all the disease.

During the laparoscopic phase, first, both ureters were identified and isolated by a retroperitoneal approach at the point where they crossed the uterine vessels; then the paravesical space, medial and lateral Okabayashi's and Latzko's pararectal spaces [21, 22] were developed. Subsequently, retrorectal (Heald's holy plane) [23] and rectosacral spaces (Waldeyer space) [24] were developed by blunt dissection up to the level of the rectosacral-fascia and rectal pillars. After identifying the sympathetic superior hypogastric plexus and pelvic splanchnic nerves, the hypogastric nerves were isolated and then radical extirpation of all the pelvic disease was performed using our consolidated nerve-sparing technique $[25,26]$. For prevention of pelvic adhesions, temporary uterine and ovarian suspensions were performed with polypropylene-0 monofilament sutures, and both stitches were knotted extracorporeally.

Then, a full-thickness discoid rectosigmoid resection was performed with the assistance of the general surgeon, according to our previously reported series [27]. For the upper-abdominal phase a median xifoumbilical incision was made instead of bilateral subcostal incisions, thereby obtaining adequate exposure with a Bookwalter self-retaining retractor [28].

\section{Upper abdominal phase}

\section{Liver mobilization}

In order to allow full diaphragmatic inspection and a more radical approach to the diaphragm, considering the extensive muscular involvement, the same surgical steps used in right upper abdominal oncological surgery were performed. The first was mobilization of the liver from the diaphragm and its ligaments, leaving only the areolar tissue surrounding the caval wall (bare area) anteriorly. The round ligament of the liver and the membranous portion of the falciform ligament were cauterized and transected, freeing the liver and anterior abdominal wall. Thus, the right triangular ligament was cut in order to access the coronal ligament and finally the right adrenal gutter. The left triangular ligament was cut, exposing the supra-hepatic veins and finally the caval ligaments and the anterior wall of the inferior vena cava. Adhesions between the liver and diaphragm were then dissected. More than two thirds of the right diaphragmatic peritoneum and more than one third of the left diaphragm contained nodules of endometriosis that occupied the full thickness of the diaphragm.

\section{Diaphragmatic resection}

Resection of the left and right diaphragm was performed. The pleural cavity was opened, revealing multiple nodules involving the parietal pleura and the diaphragmatic central tendon and infiltrating through the diaphragm to the pericardium.

A wide area of the right diaphragm and right parietal basal pleura was resected as well as wide resection of the left diaphragm, left parietal basal pleura and more than $4 \mathrm{~cm}$ of the parietal pericardium (at the level of its insertion on the central tendon). A pericardial window was created, thereby sparing the left phrenic nerve fibres (nerve-sparing wide diaphragmatic resection). No arrhythmias occurred during surgery.

Finally, a few remaining superficial (less than $3 \mathrm{~mm}$ ) diaphragmatic implants, were bilaterally cauterized with the Argon-Beam Coagulator (ABC) (Conmed Corp, Utica, NY), in order to avoid wider muscular resections. In order to assess the thoroughness of the procedure and the status of the diaphragm, lungs, heart, pleura and pericardium, a $10 \mathrm{~mm}$ laparoscope was introduced into the pleural and pericardial cavities, which demonstrated that all the disease had been removed.

\section{Pericardial, pleural and diaphragmatic reconstruction}

The pericardial window was then closed with a tension-free single layer non-absorbable suture (1-0 polypropylene). No pericardial patches were necessary, due to the good compliance of the pericardial tissue. Because of the elasticity of the tissue and the mobilization of the patient's diaphragm, it was not necessary to fill the diaphragmatic gap with artificial mesh. Accordingly, a double layer non-absorbable running suture (1-0 polypropylene) was performed in each side. As an alternative to chest tube placement for pneumothorax, a 14-Fr Foley catheter was placed directly into the pleural cavity through the partially closed diaphragmatic defect. Closure stitches were placed on either side of the catheter but not tied.

The anaesthesiologist performed several large-volume ventilations and a Valsalva manoeuvre was initi- 
ated while gentle suction was maintained in the Foley catheter. The catheter was quickly removed as the remaining closure sutures were drawn taught and tied. The absence of microscopic diaphragmatic defects was then assessed with a pneumatic test by filling the repaired diaphragmatic vaults with saline solution. Air bubbles were not detected. Drains were left, in the pelvis behind the bowel anastomosis and in Morison's pouch, which were removed on postoperative day 2. The estimated blood loss was $900 \mathrm{ml}$ and the operative time was $360 \mathrm{~min}$. The laparoscopic phase lasted $150 \mathrm{~min}$, while the upper abdominal operative time lasted $210 \mathrm{~min}$. The patient remained in the Intensive Care Unit for the post-operative night, and was transfused with a unit of red blood cells. The postoperative course was uneventful and lasted 8 days. The bladder catheter was removed on day 1 .

The patient monitored her bladder residuals after spontaneous voiding by self-catheterization for 1 day, until the urinary residual was less than $100 \mathrm{ml}$ on three consecutive essays. A chest $X$-ray on the first postoperative day showed a bilateral pneumothorax and/or hydrothorax, and a subsequent computed tomography (CT) scan confirmed mild apical pneumothorax and areas of atelectasis, particularly on the left lower lung lobe. The chest X-rays at day 3, 6 and 8 and a thoracic CT scan at day 5 showed a progressively decreasing pneumothorax with reduction of the hydrothorax and basal atelectasis.

Prophylactic antibiotic therapy with ampicillin was administered for 7 days. There were no postoperative pericardial effusions. The patient had respiratory rehabilitation for 2 weeks after discharge.

\section{Pathology report}

On gross examination the right diaphragmatic specimen measured $9 \mathrm{~cm}$ and the left diaphragmatic, pleural and pericardial nodules measured $5 \mathrm{~cm}$. The musculature of the diaphragm was replaced by several cysts filled with chocolate-like material (Figure 2). The pleural-pericardial nodule was firm and white. The diaphragmatic peritoneum, muscular layer, parietal pleura and pericardium were completely fused into one solid mass.

Histologically, the endometriotic foci were characterized by endometrial glands surrounded by endometrial stroma with foci of haemorrhage and fibroblastic proliferation. Hemosiderin deposition was present. The immunohistochemistry staining for
CD10 confirmed the endometriotic nature of the stroma $[29,30]$.

\section{Follow-up}

The patient was given medical therapy for 3 months using a GnRH-analogue (Decapeptyl $3.75 \mathrm{mg}$ ), followed by continuative oral oestrogen and progestogen, as is routinely suggested after surgery in patients with deep endometriosis [31, 32].

At 1 month and 3 and 6 months after surgery a pelvic examination was performed and a simple questionnaire was administered to determine her well-being and whether there were any complications. At 1-month follow-up the patient had no complications after discharge and no respiratory distress. Chest X-ray was normal. Echocardiogram was normal, revealing at late follow-up no pericardial effusion, and normal diastolic filling pattern. Neither rectal stenosis nor sub-stenosis was detected and there was good rectal motility (one evacuation every 1-2 days). At 3- and 6-months follow-up the patient reported no respiratory distress.

The QOL (quality of life) questionnaires for bowel, bladder and sexual function were used. Patient satisfaction about outcome of surgery was scored from 0 to 10 on the questionnaire. A score of 0-4 was considered "dissatisfied", 5-8 was "satisfied", and 9-10 was "very satisfied". She was "satisfied" after 1 month and "very satisfied" after 3, without sequelae on bowel, bladder and thoracic functions She did not experience pelvic and/or thoracic pain (VAS score = 1 for all parameters) [20].

\section{Discussion}

To the best of our knowledge, this is the only case in the literature of diaphragmatic endometriosis involving parietal and visceral pleurae and the pericardial leaf. So far, no paper has described histologically proven endometriosis of the pericardium. In fact, a review of the literature about pericardial endometriosis reports only 2 cases of radiological suspicion of pericardial endometriosis. The first is a report by Crescini et al. about a woman with tubo-ovarian endometriosis who, 50 days after laparoscopy, developed massive ascites, bilateral pleural and pericardial effusion. At re-laparotomy pelvic peritonitis was present and ascitic, pleural and pericardial fluid was secondary to superinfection, and there was no cytological or histological confirmation of endometriosis [33]. 

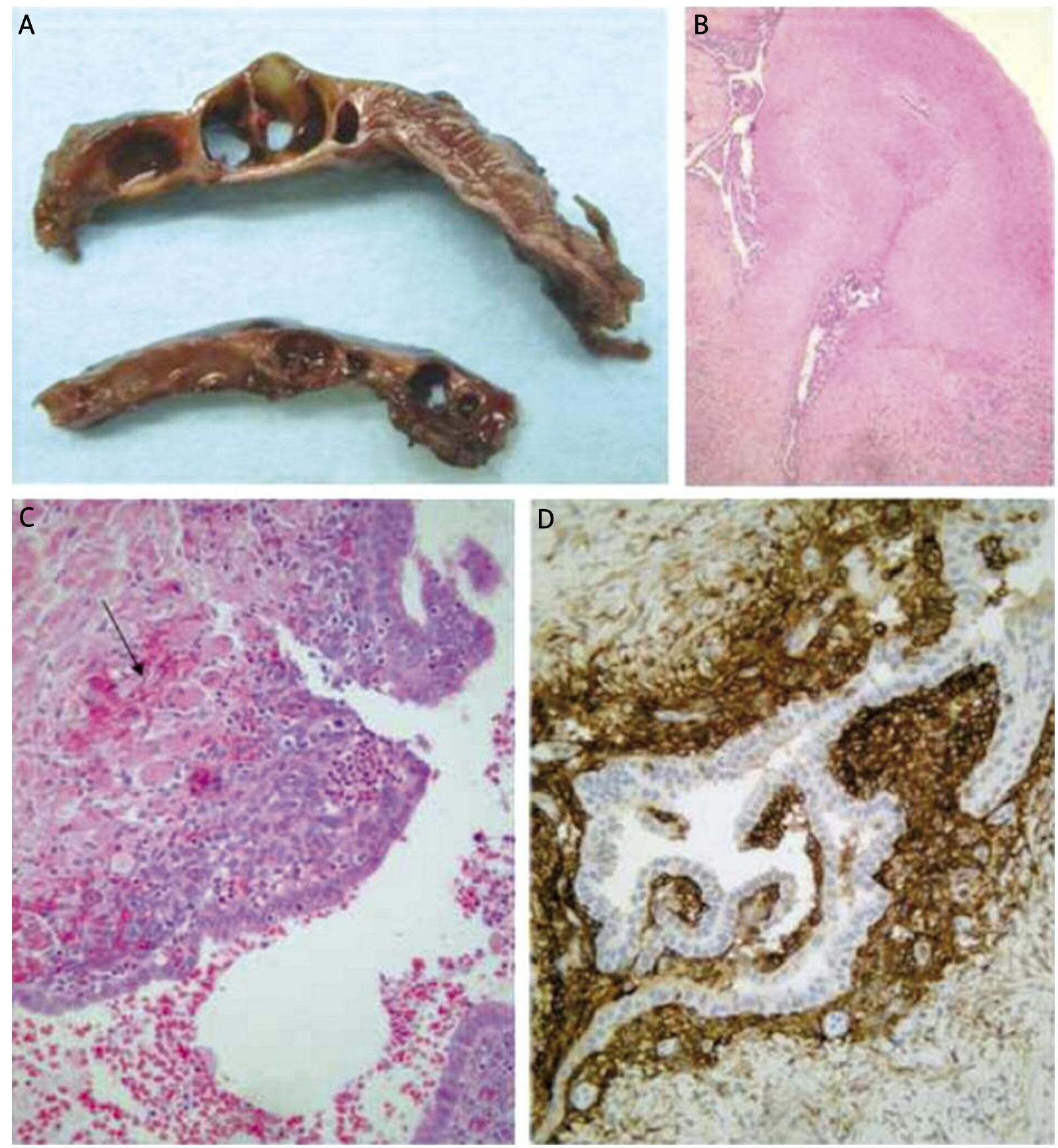

Figure 2. A - Gross section of the diaphragm. The normal architecture is destroyed by the presence of numerous endometriotic cysts. B - Histology of the parietal pericardium. The fibrous tissue is infiltrated by typical endometriosis. C - At high power a focus of endometriosis showing part of an endometriotic gland and a cuff of periglandular endometriotic stroma containing dilatated blood vessel. At the periphery myocytes are visible (arrow). D - The periglandular endometriotic stroma shows immunoreactivity for CD10

The second case was diagnosed only by computed tomography without surgical treatment and histological confirmation [34]. An experimental study in dogs reported the development of endometrial tis- sue after surgical implantation in the pericardium and diethylstilbestrol hormonal stimulation; however, the final scope of this report was to assess whether endometrium growing in this ectopic loca- 
tion might result in an increased blood supply to the heart [35].

Diaphragmatic endometriosis is a rare condition, with a specific surgical indication. It occurs mostly in women with severe pelvic endometriosis (90\%) [37], as in this case.

This consideration may be related to the primary theory of the pathogenesis of endometriosis, for which the retrograde "regurgitation" of endometrial cells passes through the oviducts into the peritoneal cavity and proliferates in ectopic sites [38]; this demonstrates the rare diaphragmatic involvement by endometriosis, and, as a consequence, the exceptionality of this case, with whole thickness bilateral substitution of the muscular layer to the parietal pleura and the pericardial leaf. The asymmetric distribution of ectopic endometrium in the diaphragm as described by Vercellini et al. [36] is confirmed in this case. This condition describes the presence of more dense endometrial implants in the right hemidiaphragm than in the left side, in accordance with the transportation of viable cells in peritoneal fluid following a clockwise circulation pattern from the pelvis up the right gutter to the right hemidiaphragm; the cells are then blocked by the falciform ligament, and over time infiltrate the right half of the muscle then the left side.

The pathogenesis of the thoracic syndrome involves compromised diaphragmatic integrity, either by endometrial implants on the surface of the diaphragm or by congenital and acquired defects which are presumed to facilitate access to the thoracic cavity. These diaphragmatic endometrial implants create channels through the muscle that allow further implants to move into the chest, leading to spontaneous pneumothorax during menses [40].

The induction theory holds that endometriosis develops from metaplasia of cells lining the pelvic peritoneum [39]. As both pleura and peritoneum develop from coelomic epithelium, it cannot be excluded that diaphragmatic endometriosis originates from coelomic metaplasia. Unknown intrinsic embryogenetic processes of the coelomic epithelium may underlie the unequal distribution of endometriosis behind apparent anatomical and physiological explanations. Moreover, if the falciform ligament interrupts the flow of endometrial cells, diaphragmatic lesions would be expected to occur clustered along the ligament, rather than distributed along the posterior margin of the diaphragm. Despite this, the observation of equal proportions of endometriosis on the right and left diaphragmatic leafs would have rendered plausible this possibility of coelomic metaplasia, but the finding of a $3: 1$ ratio in the pattern of lateral distribution of lesions [19] argues against it.

Finally, endometrial implants can metastatically spread through the uterine veins in the venous system, thus reaching the lung parenchyma and causing focal defects on the pleural surface during menstruation, leading to air leaks [41]. The diagnosis of diaphragmatic endometriosis before surgical exploration is quite difficult, firstly because of the high percentage of asymptomatic patients, then because imaging techniques lack sufficient sensitivity [42]. The $X$-ray may reveal pleural effusion or nodular opacities, which are however aspecific findings, but often it is normal. Chest computed tomography and magnetic resonance can show opacities, nodular lesions, thinwalled cavities and bullous formations [43].

Women should be asked about pleuritic, shoulder, or upper abdominal pain occurring with menses because they may not report symptoms in these distant anatomical landmarks. Catamenial pneumothorax is the most common manifestation of diaphragmatic endometriosis in cases reported in the literature [44], and this probably represents the main reason why women with this symptom are more commonly referred to a cardiothoracic specialist than to a gynaecologic surgeon. An incidental finding of endometriosis involving the diaphragm needs to be managed by an experienced surgeon, who must be familiar with the treatment of deeply infiltrating endometriotic lesions, such as a gynaecological-oncological surgeon, who usually performs upper-abdominal surgery as often required for ovarian cancer. As can be easily shown in this case and in our series, endometriosis has in fact an "onco-mimetic nature", which reflects in many aspects the clinico pathological features of ovarian cancer [25-27, 45-48].

The pelvic phase can be safely performed by the laparoscopic route, avoiding an umbilico-pubic laparotomy and allowing a radical but less invasive surgical approach, thus decreasing the length of the laparotomic phase, with lesser bowel manipulation and abdominal wall retraction.

At our institution, in the last 7 years, 789 laparoscopic bowel resections for 307 severe endometrioses have been performed [47, 25-27, 45-48]. In this 
case, the radical excision of deep pelvic endometriosis with rectosigmoid discoid resection performed laparoscopically in the first surgical phase resulted in shorter recovery, reduced hospital stay (our patient was discharged after only 8 days) and optimal aesthetic effect. Some specific aspects are fundamental to approach diaphragmatic surgery.

In the case of symptomatic patients, video-assisted thoracoscopy is the invasive method of choice and it is able to find diaphragm perforations, nodular or plaque-like, brown or violet endometrial deposits, usually less than $1 \mathrm{~cm}$ in size [49]. In the case of incidental finding during laparoscopy, the most significant endometriotic lesions are usually located at the junction of the diaphragm with the posterior edge of the liver, more frequently on the right side, and cannot be seen easily with a laparoscope from an umbilical port. Such posterior lesions can always be seen with a laparoscope inserted beneath the costal margin and with the operating table in steep reverse Trendelenburg position. However, the left diaphragm is always well visualized as it is smaller than its right counterpart.

Technical considerations may make laparoscopy a difficult approach for symptomatic diaphragmatic endometriosis. As the more symptomatic lesions are located posteriorly, the operating space is small and narrow, and additional port sites could damage the anterior diaphragm. Moreover, retraction of the liver may damage this organ. Then, as diaphragm thickness ranges between $1 \mathrm{~mm}$ and $5 \mathrm{~mm}$, aggressive laparoscopic treatment could result in a full-thickness defect of the muscle with difficulties in repair and possible herniation of the small bowel and other abdominal organs into the thorax [50].

Symptomatic diaphragmatic endometriosis is associated with lesions which are deep and which can involve essentially the entire thickness of the diaphragm. When the lesions are deep, treatment is more difficult, pain relief may not be complete, and there is a higher possibility of recurrence.

Moreover, the use of electrosurgery is hazardous when the lesions are located directly below the heart, because this may induce arrhythmias [19]. In a series of cases from Redwine [50], full-thickness resection of the diaphragm and repair performed at laparotomy resulted in an improvement of symptoms in $100 \%$ of patients and a complete cure of symptoms in $88 \%$ of patients without postoperative medical therapy.
Adequate exposure of the upper abdominal quadrants can be obtained with a midline incision by an autostatic self-retaining rectractor such as Bookwalter [28], which we used in the case described. In fact, the operation field was optimal, without obstacles to the surgical procedures.

Surgical treatment is indicated for symptomatic patients who are unresponsive to or unable to tolerate medical suppression, and for asymptomatic patients where there is concern over possible progression of the diaphragmatic lesions. Adequate counselling is needed regarding the possible risks associated with the treatment of extensive endometriosis that involves different organs. Before surgery, the possibility of pleural or pulmonary involvement should be investigated according to the associated symptoms. A cardiopulmonary consultant should evaluate the patient and the support of a thoracic surgical team must be available [19].

Comprehensive knowledge of the anatomy of the diaphragm and of the surgical steps and techniques of diaphragmatic surgery are needed in order to avoid intra- and post-operative morbidity such as pneumothorax and subsequent hypoventilation and dyspnea.

Guidelines for the treatment of diaphragmatic endometriosis proposed by Nezhat indicate that a safe surgical procedure is possible when the endometriotic lesions are superficial and located at an adequate distance from the left ventricle or the phrenic nerve [19].

The right phrenic nerve penetrates the central tendon of the diaphragm lateral to the vena caval foramen on the right and is usually not encountered until the "bare area" is exposed. The left phrenic nerve may penetrate the left diaphragm muscle above the central tendon and is a consideration during left-sided anterior diaphragm surgery.

The aetiology of diaphragm paralysis is variable [51] and can be traumatic, compression-related, inflammatory, neuropathic and idiopathic. The traumatic causes of diaphragm paralysis include openheart surgery, lung transplantation, oesophageal surgery and mediastinal procedures. The left diaphragm is usually more commonly involved than the right diaphragm. However, the prognosis in unilateral paralysis is usually good, especially in the absence of underlying lung disease.

Compensatory mechanisms usually kick in after acute paralysis and support ventilation. If the injury is 
minor or distal, phrenic nerve function improves with time, with restoration of diaphragmatic function.

Although reports of identification of the phrenic nerve at laparoscopy have been offered by Nezhat's group [19], this appears unlikely. In the present case report, it cannot be identified as a discrete structure on either side of the diaphragm. Resection of large portions of the diaphragm might raise concern about injury to this nerve with resultant dysfunction of the diaphragm, but in our case this did not seem to be a clinically important issue.

Although surgical specimens ranged up to $9 \mathrm{~cm}$ in maximum dimension, the diaphragm could be easily closed in each patient without tension on the suture line. Such an incision line follows the ancient surgical axiom that "healing occurs from side to side, not end to end." Artificial mesh was not used to repair the diaphragm because of the possible heavy sequelae in case of faecal peritonitis due to dehiscence or fistula of the bowel anastomosis.

As described in our case report, we think that a Foley or Robinson catheter, directly introduced into the pleural cavity and quickly removed by gentle aspiration during maximal lung expansion, should be a standard technique to avoid unnecessary thoracic drains, which can cause thoracic pain and limit respiratory excursions in the post-operative rehabilitation period, as well as being a possible cause of infection.

\section{Conclusions}

This case report underlines the importance of surgical expertise for the treatment of severe endometriosis, where comprehensive knowledge of radical and oncological surgical techniques and anatomy is required to manage unexpected findings such as diaphragmatic, pleural or even pericardial endometriosis. Treatment of diaphragmatic endometriosis must be individualized on the basis of the patient's age, desires for fertility, extent and localization of lesions and symptomatology.

Laparoscopy is supported by the literature for pelvic disease, whereas for diaphragmatic endometriosis it results in a high rate of incomplete diagnosis and treatment with a high rate of continuing symptoms. Adequate visualization of the posterior right diaphragm is mandatory and can be achieved by a laparoscope inserted beneath the costal margin and with the patient in reverse Trendelenburg position; however, in the case of symp- tomatic deep-infiltrating nodules, abdominal surgery is recommended but with respect of anatomical structures.

Extensive experience in upper abdominal surgery - such as for the treatment of ovarian cancer - and the support of a basic knowledge of thoracic surgery are the cornerstones for the approach of this rare condition, in order to avoid complications and to perform a safe but complete resection of the disease with optimal relief of symptoms.

\section{Acknowledgments}

The authors would like to acknowledge Prof. Robert J. Kurman (from the Department of Pathology, Johns Hopkins Medical Institutions, Baltimore, MD 21231, USA) for paper revision and precious technical and scientific suggestions.

\section{References}

1. Rock JA, Markham SM. Extra pelvic endometriosis. In: Endometriosis. Wilson EA (ed.). New York, Alan Liss, 1987; 185-206.

2. Giudice LC, Tazuke SI, Swiersz L. Status of current research on endometriosis. J Reprod Med 1998; 43 (3 Suppl): 252-62.

3. Jubanyik KJ, Comite F. Extrapelvic endometriosis. Obstet Gynecol Clin North Am 1997; 24: 411-40.

4. Kumar V, Cotran RS, Robbins SL. Female genital system and breast. In: Basic pathology. Kumar V, Cotran RS, Robbins SL (eds.). Ed. 6. Philadelphia, Saunders 1997; chapter 19: 608-9.

5. Sensenig DM, Serlin O, Hawthorne HR. Pericardial endometriosis. An experimental study in dogs. JAMA 1966; 198: 645-7.

6. Martin JD Jr, Haucl AE. Endometriosis in the male. Am Surg 1985; 51: 426-30.

7. Schrodt Gr, Alcorn MO, Ibanez J. Endometriosis of the male urinary system. J Urol 1980; 124: 722-3.

8. Posniak H, Keshavarzian A, Jabamoni R. Diaphragmatic endometriosis: CT and MR findings. Gastrointest Radiol 1990; 15 : 349-51.

9. Nezhat F, Nezhat C, Levy JS. Laparoscopic treatment of symptomatic diaphragmatic endometriosis: a case report. Fertil Steril 1992; 58: 614-6.

10. Chinegwundoh F, Ryan P, Luesley T, Chan SY. Renal and diaphragmatic endometriosis de novo associated with hormone replacement therapy. J Urol 1995; 153: 380-1.

11. Witte A, Guilbaud O. Endometriose du diaphragme. Aspectc diagnostiques a propos d'un cas sans pneumothorax. Rev Med Interne 1995; 16: 527-32.

12. Mangal R, Taskin O, Nezhat C, Franklin R. Laparoscopic visualization of diaphragmatic endometriosis in a woman with epigastric pain: a case report. J Reprod Med 1996; 41: 64-6.

13. Cooper MJW, Russel P, Gallagher PJ. Diaphragmatic endometriosis. Med J Aus 1999; 171: 142-3.

14. Wolthuis AM, Aelvoet C, Bosteels J, Vanrijkel JP. Diaphragmatic endometriosis: diagnosis and surgical management - a case report. Acta Chir Belg 2003; 103: 519-20. 
15. Nahir Eldar-Geva T, Alberton J, Beller U. Symptomatic diaphragmatic endometriosis ten years after total abdominal hysterectomy. Obstet Gynecol 2004; 104: 1149-51.

16. Takeuchi H, Kitade M, Sakurai A, et al. Fitz-Hugh and Curtis syndrome-like diaphragmatic endometriosis. Fertil Steril 2005; 83: 1039-40.

17. Cucinella G, Granese R, Calagna G, et al. Laparoscopic treatment of diaphragmatic endometriosis causing chronic shoulder and arm pain. Acta Obstet Gynecol Scand 2009; 88: 1418-9.

18. Gilalbert-Estelles J, Zorio E, Castello JM, et al. Laparoscopic approach to right diaphragmatic endometriosis with argon laser: case report. J Minim Invasive Gynecol 2010; 17: 124-7.

19. Nezhat C, Seidman DS, Nezhat F, Nezhat C. Laparoscopic surgical management of diaphragmatic endometriosis. Fertil Steril 1998; 69: 1048-55.

20. Wewers ME, Lowe NK. A critical review of visual analogue scales in the measurement of clinical phenomena. Res Nursing Health 1990; 13: 227-36.

21. Okabayashi H. Radical abdominal hysterectomy for cancer of the cervix uteri, modification of the Takayama operation. Surg Gynecol Obstet 1921; 33: 335-41.

22. Peham HV, Amreich J. Gyna" kologische Operationslehre. Karger, Berlin 1930; 350-85.

23. Heald RJ. The 'Holy Plane' of rectal surgery. J R Soc Med 1988; 81: 503-8.

24. Waldeyer W. Das Becken. Cohen, Bonn 1899.

25. Ceccaroni M, Pontrelli G, Scioscia M, et al. Nerve-sparing laparoscopic radical excision of deep endometriosis with rectal and parametrial resection. J Minimal Invasive Gynecol 2010; 17: 14-5.

26. Landi S, Ceccaroni M, Perutelli A, et al. Laparoscopic nerve-sparing complete excision of deep endometriosis: is it feasible? Hum Reprod 2006; 21: 774-81.

27. Fanfani F, Fagotti A, Gagliardi ML, et al. Discoid or segmental resection for deep infiltrating endometriosis: a case-control study. Fertil Steril 2010; 94: 444-9.

28. Del Carmen MG, Bristow RE, Duska LR. Surgical instrumentation - retractors. In: Surgery for ovarian cancer - principles and practice. Bristow RE, Karlan BY (eds.). Taylor \& Francis 2007; 47.

29. Nucci MR, Oliva E. Gynecological pathology. Foundation in Diagnostic Pathology. Churchill Livingstone 2009; chapter 15: 615-21.

30. Colella R, Mameli MG, Bellezza G, et al. Endometriosis-associated skeletal muscle regeneration: a hitherto undescribed entity and a potential diagnostic pitfall. Am J Surg Path 2010; 34: 10-17.

31. Vercellini P, Pietropaolo G, De Giorgi O, et al. Treatment of symptomatic rectovaginal endometriosis with an estrogen-progestogen combination versus low dose norethindrone acetate. Fertil Steril 2005; 84: 1375-87.

32. Vercellini P, Crosignani PG, Somigliana E, et al. Medical treatment for rectovaginal endometriosis: what is the evidence? Hum Reprod 2009; 24: 2504-14.

33. Crescini C, von Wustner S, Spazzini D. Ascites and pleural and pericardial effusion after operative laparoscopy. J Am Assoc Gynecol Laparosc 2000; 7: 155-7.

34. Francis $M$, et al. Pericardial effusion, right-sided pleural effusion and ascites associated with stage IV endometriosis. J Reprod Med 2003; 48: 463-5.

35. Sensenig DM, Serlin O, Hawthorne HR. Pericardial endometriosis. An experimental study in dogs. JAMA 1966; 198: 645-7.
36. Vercellini $\mathrm{P}$, Abbiati A, Viganò $\mathrm{P}$, et al. Asymmetry in distribution of diaphragmatic endometriotic lesions: evidence in favour of the menstrual reflux theory. Hum Reprod 2007; 22: 2359-67.

37. Foster DC, Stern JL, Buscema J, Rock JA, Woodruff JD. Pleural and endometrial endometriosis. Obstet Gynecol 1981; 58: 552-6.

38. Sampson J. Peritoneal endometriosis due to dissemination of endometrial tissue into the peritoneal cavity. Am J Obstet Gynecol 1927; 14: 422.

39. Russell P, Farnsworth A. Mullerianosis and reactive mesothelial lesions. Surgical pathology of the ovaries. Churchill Livingstone, New York 1997; 195-207.

40. Foster DC, Stern JL, Buscema J, et al. Pleural and parenchymal pulmonary endometriosis. Obstet Gynecol 1981; 58: 552-6.

41. Shearin RPN, Hepper NGG, Payne WS. Recurrent spontaneous pneumothorax concurrent with menses. Mayo Clinic Proc 1974; 49: 98-101.

42. Witte A, Goulband O. Endometriosis of the diaphragm. Diagnostic aspects apropos of a case without pneumothorax. Rev Med Interne 1995; 16: 527-32.

43. Posniak HV, Keshavarzian A, Jabamoni R. Diaphragmatic endometriosis: CT and MR findings. Gastrointest Radiol 1990; 15: 349-51.

44. Korom S, Canyurt H, Missbach A et al. Catamenial pneumothorax revisited: clinical approach and systematic review of the literature. J Thorac Cardiovasc Surg 2004; 128: 502-8.

45. Minelli L, Fanfani F, Fagotti A, et al. Laparoscopic colorectal resection for bowel endometriosis: feasibility, complications, and clinical outcome. Arch Surg 2009; 144: 234-9.

46. Minelli L, Ceccaroni M, Ruffo G, et al. Laparoscopic conservative surgery for stage IV symptomatic endometriosis: short-term surgical complications. Fertil Steril 2009, Dec 5 [Epub ahead of print].

47. Ruffo G, Scopelliti F, Scioscia M, et al. Laparoscopic colorectal resection for deep infiltrating endometriosis: analysis of 436 cases. Surg Endosc 2010; 24: 63-7.

48. Stepniewska A, Pomini P, Bruni F, et al. Laparoscopic treatment of bowel endometriosis in infertile women. Hum Reprod 2009; 24: $1619-25$

49. Tsunezuka Y, Oda M, Moriyama H, et al. Thorascopic findings and surgical management of catamenial hemopneumothorax. Ann Thorac Cardiovasc Surg 2006; 12: 197-9.

50. Redwine DB. Diaphragmatic endometriosis: diagnosis, surgical management, and long-term results of treatment. Fertil Steril 2002; 77: 288-96.

51. Qureshi A. Diaphragm paralysis. Semin Respir Crit Care Med 2009; 30: 315-20. 